\title{
A scalable synthesis of the (S)-4-(tert-butyl)-2-(pyridin-2-yl)-4,5-dihydrooxazole ((S)-t-BuPyOx) ligand
}

\author{
Hideki Shimizu ${ }^{1,2}$, Jeffrey C. Holder ${ }^{1}$ and Brian M. Stoltz ${ }^{* 1}$
}

\section{Full Research Paper}

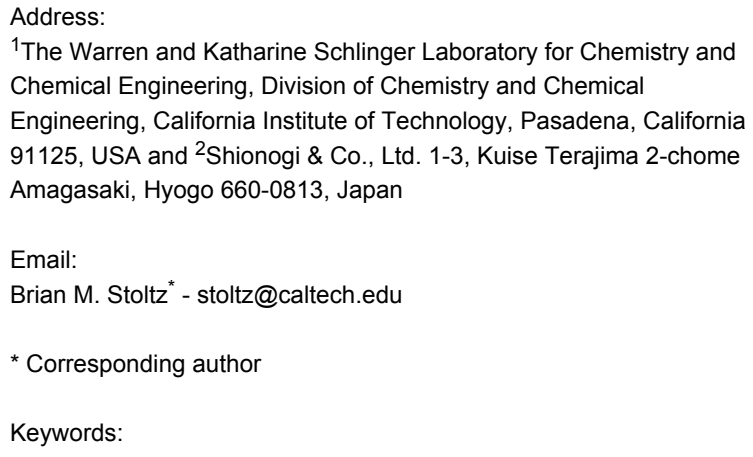

${ }^{1}$ The Warren and Katharine Schlinger Laboratory for Chemistry and Chemical Engineering, Division of Chemistry and Chemical Engineering, California Institute of Technology, Pasadena, California 91125, USA and ${ }^{2}$ Shionogi \& Co., Ltd. 1-3, Kuise Terajima 2-chome Amagasaki, Hyogo 660-0813, Japan

Email:

Brian M. Stoltz* - stoltz@caltech.edu

${ }^{*}$ Corresponding author

Keywords:

asymmetric catalysis; diamine ligands; optimization; synthesis

\author{
Beilstein J. Org. Chem. 2013, 9, 1637-1642. \\ doi:10.3762/bjoc.9.187 \\ Received: 06 June 2013 \\ Accepted: 15 July 2013 \\ Published: 12 August 2013 \\ Associate Editor: J. P. Wolfe \\ (c) 2013 Shimizu et al; licensee Beilstein-Institut. \\ License and terms: see end of document.
}

\begin{abstract}
An efficient method for the synthesis of the (S)-4-(tert-butyl)-2-(pyridin-2-yl)-4,5-dihydrooxazole ((S)-t-BuPyOx) ligand has been developed. Inconsistent yields and tedious purification in known routes to $(S)$ - $t$-BuPyOx suggested the need for an efficient, dependable, and scalable synthetic route. Furthermore, a route suitable for the synthesis of PyOx derivatives is desirable. Herein, we describe the development of a three-step route from inexpensive and commercially available picolinic acid. This short procedure is amenable to multi-gram scale synthesis and provides the target ligand in $64 \%$ overall yield.
\end{abstract}

\section{Introduction}

Pyridinooxazoline (PyOx) ligands represent a growing class of bidentate dinitrogen ligands used in asymmetric catalysis [1-23]. Recently, our laboratory reported the catalytic asymmetric conjugate addition of arylboronic acids to cyclic, $\beta, \beta$-disubstituted enones utilizing $(S)$ - $t$-BuPyOx (1) as the chiral ligand (Figure 1) [24]. This robust reaction is insensitive to oxygen atmosphere, highly tolerant of water [25], and provides cyclic ketones bearing $\beta$-benzylic quaternary stereocenters in high yields and enantioselectivities. While the reaction itself proved to be amenable to multi-gram scale, the ligand is not yet commercially available and no reliable method for the large- scale synthesis of $(S)$ - $t$-BuPyOx was known (a number of syntheses are known, including [26]). We sought to address this shortcoming by developing an efficient route starting from a cheap, commercially available precursor to pyridinooxazoline ligands. Herein, we report an efficient, highly scalable synthesis of $(S)$ - $t$-BuPyOx.

\section{Results and Discussion}

Initially, $(S)$-t-BuPyOx (1) was synthesized by methanolysis of 2-cyanopyridine (2) to afford methoxyimidate $\mathbf{3}$, and subsequent acid-catalyzed cyclization to afford the $(S)$ - $t$-BuPyOx 


\section{Asymmetric conjugate addition with ( $S$ )- $t$-BuPyOx}<smiles>[R]C1=CC(=O)CCC1</smiles>

Initial route to (S)-t-BuPyOx:

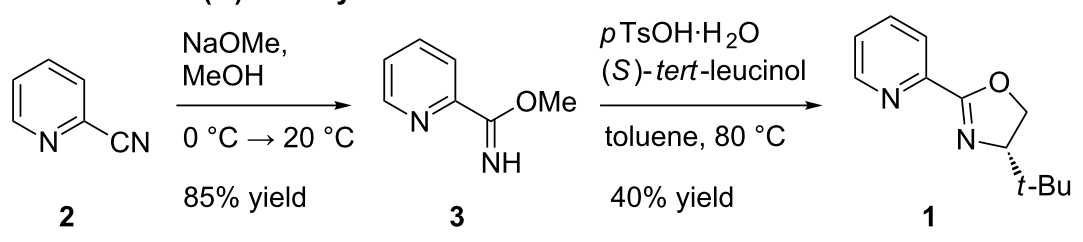

Revised retrosynthetic plan:<smiles>CC(C)(C)[C@H]1COC(c2ccccn2)=N1</smiles>

1<smiles>C=Cc1cccc(C(=O)NC(CO)[C@@H](CC=C=Cc2cccc(C(=O)O)n2)C(C)(C)C)n1</smiles>

5
6

Figure 1: Initial PyOx synthesis and revised plan.

ligand (Figure 1) [27]. We found the yields of this reaction sequence to be highly variable, and the purification by silica gel chromatography to be tedious. In the revised retrosynthesis, picolinic acid (5) was identified as a comparably priced, commonly available surrogate for cyanopyridine 2. Amidation of (S)-tert-leucinol (6) and picolinic acid (5) would generate amide 4 , which upon cyclization would generate the ligand framework.

Initial efforts focused on the amidation reaction between (S)-tert-leucinol and picolinic acid (5) via acid chloride 7 (Table 1), which was generated in situ by treatment of acid 5 with a number of chlorinating agents. Oxalyl chloride (Table 1, entries 1,2 ) provided reasonable yields of amide $\mathbf{4}$, however bisacylation of $(S)$-tert-leucinol was observed as a common side product. Importantly, temperature control of this reaction (Table 1, entry 2) allowed the isolation of $75 \%$ of desired alcohol 4 in acceptable purity without the use of column chromatography. Use of diphenyl chlorophosphate (Table 1, entries $3,5,6)$ also resulted in noticeable quantities of over-acylation products, as well as the generation of a small amount of phosphorylation of amide 4 . These results encouraged us to explore alternative activation strategies to generate the desired amide bond. Adapting a procedure from Sigman, activation of acid 5 by treatment with isobutylchloroformate and $\mathrm{N}$-methylmorpholine (anhydride 8) facilitated the desired transformation with the highest overall yield, with amide 4 being isolated in $92 \%$ yield, albeit requiring column chromatography [28].
Satisfied with our ability to generate amide $\mathbf{4}$ on gram-scale with good yield, we turned our attention to the completion of the synthesis. The cyclization of amide 4 to $(S)$ - $t$-BuPyOx (1) proved more challenging than anticipated. Activation of alcohol 4 as mesylate 9 (Table 2, entries 1,2) and tosylate $\mathbf{1 0}$ (Table 2, entry 3 ) followed by in situ cyclization gave the desired product in low yield and incomplete conversion. This could potentially result from ligand hydrolysis under the given reaction conditions [29]. As an alternative to insitu cyclization of an activated intermediate, alcohol 4 was reacted with thionyl chloride (Table 2, entries 4-10) to yield chloride 11, which was isolated as the hydrochloric acid salt and dried under vacuum. This compound proved to be bench stable and was spectroscopically unchanged after being left open to oxygen atmosphere and adventitious moisture for more than one week. Furthermore, chloride $\mathbf{1 1}$ proved to be a superior cyclization substrate. A series of bases were screened. Organic amine bases (Table 2, entries 4,5) and sodium hydride (Table 2, entry 6) provided inadequate conversion and low yields, whereas hydroxide and alkoxide bases proved superior (Table 2, entries 7-10). Finally, sodium methoxide was chosen to be optimal, as slower rates of hydrolysis of chloride $\mathbf{1 1}$ were observed when compared to the use of potassium hydroxide.

Attempts to purify ligand $\mathbf{1}$ via salt formation failed due to instability of the generated products [30]. Purification by silica gel chromatography also proved challenging as up to $10 \%$ of crude ligand 1 was observed to decompose, even with the addi- 
Table 1: Amidation reactions of picolinic acid

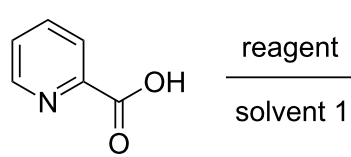

5<smiles></smiles>

$7 \mathrm{X}=\mathrm{Cl}$

$8 \mathrm{X}=\mathrm{OCO}$ iBu
(S)-tert-leucinol base

solvent 2<smiles>CCCC[C@@H](CO)NC(=O)c1ccccn1</smiles>

4

\begin{tabular}{|c|c|c|c|c|c|c|}
\hline entry & reagent & solvent $1 / 2$ & temp $\left({ }^{\circ} \mathrm{C}\right)^{\mathrm{a}}$ & base & time $(h)^{b}$ & yield $(\%)^{c}$ \\
\hline 1 & $(\mathrm{COCl})_{2}$ & THF/THF & 50 & $\mathrm{Et}_{3} \mathrm{~N}$ & 1 & 55 \\
\hline 2 & $(\mathrm{COCl})_{2}$ & THF/THF & 0 to rt & $\mathrm{Et}_{3} \mathrm{~N}$ & 7 & $75^{\mathrm{d}}$ \\
\hline 3 & DPCP & THF/THF & 0 to $r t$ & $\mathrm{Et}_{3} \mathrm{~N}$ & 6 & 72 \\
\hline 4 & $\mathrm{SOCl}_{2}$ & toluene/THF & $\mathrm{rt}$ & none & 5 & trace \\
\hline 5 & DPCP & THF/THF & 50 & none & 2 & 30 \\
\hline 6 & DPCP & THF/THF & 0 to rt & $\mathrm{Et}_{3} \mathrm{~N}$ & 3 & $65^{d}$ \\
\hline 7 & iBuOCOCl, NMM & $\mathrm{CH}_{2} \mathrm{Cl}_{2} / \mathrm{CH}_{2} \mathrm{Cl}_{2}$ & 0 to rt & NMM & 3 & 92 \\
\hline
\end{tabular}

$\mathrm{DPCP}=$ diphenyl chlorophosphate, $\mathrm{NMM}=\mathrm{N}$-methylmorpholine. ${ }^{\mathrm{a}}$ Temperature for second step; ${ }^{\mathrm{b}}$ Time for second step; ${ }^{\mathrm{C}} \mathrm{solated}$ yield; ${ }^{\mathrm{d}} \mathrm{Purification}$ by flash chromatography not required.

Table 2: Cyclization screen.

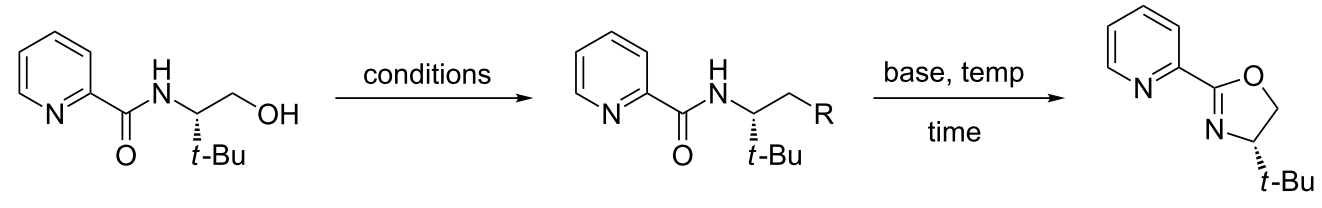

4

$9 \mathrm{R}=\mathrm{OMs}$

1

$10 \mathrm{R}=\mathrm{OTs}$

$11 \mathrm{R}=\mathrm{Cl} \cdot \mathrm{HCl}$

\begin{tabular}{|c|c|c|c|c|c|c|}
\hline entry & conditions & $\mathrm{R}$ & temp $\left({ }^{\circ} \mathrm{C}\right)$ & base & time $(h)$ & yield $(\%)^{a}$ \\
\hline 1 & $\mathrm{MsCl}, \mathrm{Et}_{3} \mathrm{~N}, \mathrm{CH}_{2} \mathrm{Cl}_{2}$ & OMs & 0 to 40 & $\mathrm{Et}_{3} \mathrm{~N}$ & 12 & N.D. ${ }^{b}$ \\
\hline 2 & $\mathrm{MsCl}, \mathrm{Et}_{3} \mathrm{~N}, \mathrm{ClCH}_{2} \mathrm{CH}_{2} \mathrm{Cl}$ & OMs & 0 to 80 & $\mathrm{Et}_{3} \mathrm{~N}$ & 12 & N.D. ${ }^{b}$ \\
\hline 3 & $\mathrm{TsCl}, \mathrm{DMAP}, \mathrm{Et}_{3} \mathrm{~N}, \mathrm{ClCH}_{2} \mathrm{CH}_{2} \mathrm{Cl}$ & OTs & 0 to 80 & $\mathrm{Et}_{3} \mathrm{~N}$ & 12 & N.D. ${ }^{b}$ \\
\hline 4 & $\mathrm{SOCl}_{2}$ & $\mathrm{Cl}^{\mathrm{C}}$ & $\mathrm{rt}$ & $\mathrm{DABCO}$ & 18 & 38 \\
\hline 5 & $\mathrm{SOCl}_{2}$ & $\mathrm{Cl}^{\mathrm{C}}$ & 50 & DBU & 12 & 59 \\
\hline 6 & $\mathrm{SOCl}_{2}$ & $\mathrm{Cl}^{\mathrm{C}}$ & 0 to 50 & $\mathrm{NaH}, \mathrm{THF}$ & 18 & 60 \\
\hline 7 & $\mathrm{SOCl}_{2}$ & $\mathrm{Cl}^{\mathrm{C}}$ & 50 & $5 \% \mathrm{KOH} / \mathrm{EtOH}$ & 11 & 58 \\
\hline 8 & $\mathrm{SOCl}_{2}$ & $\mathrm{Cl}^{\mathrm{C}}$ & 50 & $5 \% \mathrm{KOH} / \mathrm{MeOH}$ & 11 & 62 \\
\hline 9 & $\mathrm{SOCl}_{2}$ & $\mathrm{Cl}^{\mathrm{C}}$ & 50 & $25 \% \mathrm{NaOMe} / \mathrm{MeOH}$ & 10 & 71 \\
\hline 10 & $\mathrm{SOCl}_{2}$ & $\mathrm{Cl}^{\mathrm{C}}$ & 50 & $25 \% \mathrm{NaOMe} / \mathrm{MeOH}$ & 3 & 72 \\
\hline
\end{tabular}

$\mathrm{MsCl}=$ methanesulfonyl chloride, $\mathrm{TsCl}=$ 4-toluenesulfonyl chloride, $\mathrm{DMAP}=$ 4-dimethylaminopyridine, $\mathrm{DABCO}=1$,4-diazabicyclo[2.2.2]octane, $\mathrm{DBU}=$ 1,8-diazabicyclo[5.4.0]undec-7-ene. ${ }^{\text {II }}$ Isolated yield; Incomplete conversion; ' Intermediate 11 isolated as $\mathrm{HCl}$ salt and dried under high vacuum before use in cyclization reactions.

tion of triethylamine to the eluent. Finally, the use of neutral silica gel (American International Chemical ZEOprep ECO silica gel, 40-63 micron, \$18/kg) allowed isolation of ligand 1 in high purity and with no observed decomposition.

\section{Conclusion}

In conclusion, we have developed a concise, highly efficient and scalable synthesis of the chiral ligand $(S)$ - $t$-BuPyOx (1) (Figure 2). Efforts to further refine the synthesis by telescoping 


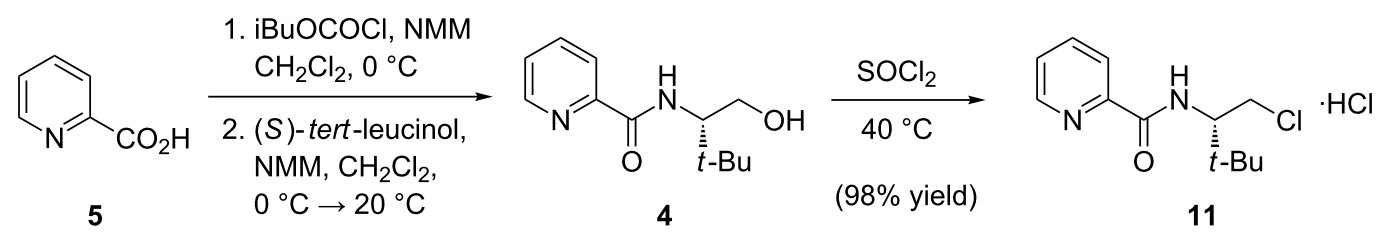

$(92 \%$ yield)

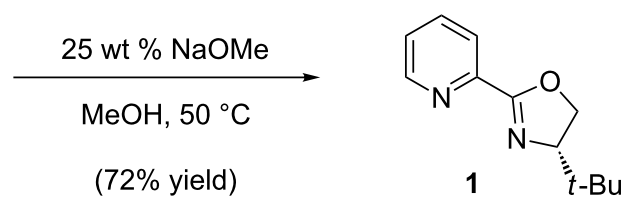

Figure 2: Scale-up synthesis of (S)-t-BuPyOx.

the procedure and removing chromatographic purifications are currently underway.

\section{Experimental}

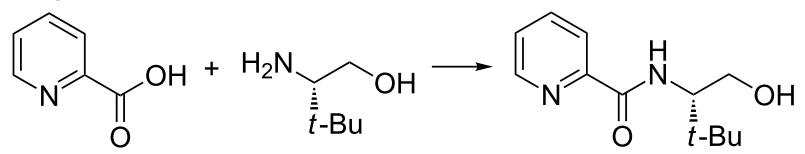

picolinic acid

5
(S)-tert-leucinol 6 amide alcohol

$$
4
$$

(S)-N-(1-hydroxy-3,3-dimethylbutan-2-yl)picolinamide (4): To a $200 \mathrm{~mL}$ round bottom flask was added picolinic acid (2.46 g, $20.0 \mathrm{mmol}, 1.00$ equiv), $50 \mathrm{~mL} \mathrm{CH}_{2} \mathrm{Cl}_{2}$, and $N$-methylmorpholine ( $3.03 \mathrm{~g}, 30.0 \mathrm{mmol}, 1.50$ equiv). The reaction mixture was cooled to $0{ }^{\circ} \mathrm{C}$ in an ice bath and isobutyl chloroformate ( $3.14 \mathrm{~g}, 23.0 \mathrm{mmol}, 1.15$ equiv) was added dropwise over $30 \mathrm{~min}$. Following complete addition, the reaction mixture was stirred for $30 \mathrm{~min}$ at $0{ }^{\circ} \mathrm{C}$. In a separate flask, $(S)$-tert-leucinol ( $2.58 \mathrm{~g}, 22.0 \mathrm{mmol}, 1.10$ equiv) was dissolved in $\mathrm{CH}_{2} \mathrm{Cl}_{2}$ $(25 \mathrm{~mL})$, and $\mathrm{N}$-methylmorpholine $(2.43 \mathrm{~g}, 24.0 \mathrm{mmol}$, 1.20 equiv) was added. This solution was transferred dropwise over the course of $1 \mathrm{~h}$ to the cooled reaction mixture using a syringe pump. The cooling bath was removed and the reaction mixture was allowed to warm to room temperature and stirred for $2 \mathrm{~h}$. The mixture was quenched with an aqueous solution of $\mathrm{NH}_{4} \mathrm{Cl}\left(10 \mathrm{~g}\right.$ in $\left.50 \mathrm{~mL} \mathrm{H} \mathrm{H}_{2} \mathrm{O}\right)$ and the aqueous phase was extracted with $\mathrm{CH}_{2} \mathrm{Cl}_{2}(20 \mathrm{~mL})$. The combined organic phase was dried over $\mathrm{Na}_{2} \mathrm{SO}_{4}(5 \mathrm{~g})$, filtered, and concentrated under reduced pressure. The residue was purified with flash silica gel column chromatography ( $4: 1$ hexanes/acetone) to afford amide alcohol 4 as a white solid $\left(4.10 \mathrm{~g}, 92 \%\right.$ yield). $R_{\mathrm{f}} 0.32$ with $3: 2$ hexanes/acetone; mp 79.6-79.9 ${ }^{\circ} \mathrm{C} ;{ }^{1} \mathrm{H}$ NMR $(500 \mathrm{MHz}$, $\left.\mathrm{CDCl}_{3}\right) \delta 8.56(\mathrm{ddd}, J=4.8,1.8,0.9 \mathrm{~Hz}, 1 \mathrm{H}), 8.32(\mathrm{br} \mathrm{d}$, $J=8.9 \mathrm{~Hz},-\mathrm{NH}), 8.19(\mathrm{dt}, J=7.8,1.1 \mathrm{~Hz}, 1 \mathrm{H}), 7.85(\mathrm{td}$, $J=7.7,1.7 \mathrm{~Hz}, 1 \mathrm{H}), 7.43(\mathrm{ddd}, J=7.6,4.8,1.2 \mathrm{~Hz}, 1 \mathrm{H})$,
4.02-3.96 (m, 2H), $3.69(\mathrm{~m}, 1 \mathrm{H}), 2.72$ (br t, $J=6.5 \mathrm{~Hz},-\mathrm{OH})$, $1.05(\mathrm{~s}, 9 \mathrm{H}) ;{ }^{13} \mathrm{C} \mathrm{NMR}\left(125 \mathrm{MHz}, \mathrm{CDCl}_{3}\right) \delta 165.6,149.7$, 148.2, 137.6, 126.4, 122.6, 63.7, 60.6, 33.9, 27.1; IR (neat film, $\mathrm{NaCl}): 3375,2962,1669,1591,1570,1528,1465,1434,1366$, 1289, 1244, 1088, 1053, $998 \mathrm{~cm}^{-1}$; HRMS (MultiMode ESI/APCI) $m / z:[\mathrm{M}+\mathrm{H}]^{+}$calcd for $\mathrm{C}_{12} \mathrm{H}_{19} \mathrm{~N}_{2} \mathrm{O}_{2}, 223.1447$; found, 223.1448; $[\alpha]^{25}{ }_{\mathrm{D}}-8.7$ (c 1.17, $\mathrm{CHCl}_{3},>99 \%$ ee).<smiles>CC(C)(C)[C@H](CO)NC(=O)c1ccccn1</smiles>

amide alcohol 4<smiles>CC(C)(C)[C@H](CCl)NC(=O)c1ccccn1</smiles>

amide chloride $\cdot \mathrm{HCl}$ 11
(S)-N-(1-chloro-3,3-dimethylbutan-2-yl)picolinamide hydrochloride (11): A $500 \mathrm{~mL} 3$-neck round bottom flask was charged with a stir bar, amide alcohol 4 (8.89 g, $40.0 \mathrm{mmol}$, 1.00 equiv) and toluene $(140 \mathrm{~mL})$. The resulting clear solution was warmed to $60{ }^{\circ} \mathrm{C}$. In a separate flask, $\mathrm{SOCl}_{2}(9.25 \mathrm{~g}$, $80.0 \mathrm{mmol}, 2.00$ equiv) was diluted with toluene $(20 \mathrm{~mL})$. This solution was transferred slowly, dropwise, over $20 \mathrm{~min}$ to the vigorously stirring reaction mixture at $60{ }^{\circ} \mathrm{C}$. The reaction mixture was stirred at $60^{\circ} \mathrm{C}$ for $4 \mathrm{~h}$, at which time the slurry was cooled to ambient temperature, concentrated on a rotary evaporator under reduced pressure $\left(40{ }^{\circ} \mathrm{C}, 40 \mathrm{mmHg}\right)$, and dried under vacuum $(0.15 \mathrm{mmHg})$ to give a white powder of amide chloride hydrochloric salt 11 (10.80 g, 98\% yield). This material was used in the following step without purification. ${ }^{1} \mathrm{H}$ NMR $\left(500 \mathrm{MHz}, \mathrm{DMSO}-d_{6}\right) \delta 8.70$ (ddd, $J=4.8,2.0$, $1.0 \mathrm{~Hz}, 1 \mathrm{H}), 8.66$ (br d, $J=9.9 \mathrm{~Hz},-\mathrm{NH}), 8.10(\mathrm{dt}, J=8.0$, $1.0 \mathrm{~Hz}, 1 \mathrm{H}), 8.06(\mathrm{td}, J=7.5,1.4 \mathrm{~Hz}, 1 \mathrm{H}), 7.66(\mathrm{ddd}, J=7.4$, $4.8,1.4 \mathrm{~Hz}, 1 \mathrm{H}), 4.08(\mathrm{td}, J=9.9,3.7 \mathrm{~Hz}, 1 \mathrm{H}), 3.97-3.90(\mathrm{~m}$, $2 \mathrm{H}), 0.93(\mathrm{~s}, 9 \mathrm{H}) ;{ }^{13} \mathrm{C}$ NMR $\left(125 \mathrm{MHz}, \mathrm{DMSO}-d_{6}\right) \delta 163.6$, $149.0,147.8,138.1,126.5,122.0,59.0,44.9,35.0,26.3$; IR (neat film, $\mathrm{NaCl}$ ): 3368, 2963, 1680, 1520, 1465, 1434, 1369, 1285, 1239, 1087, $998 \mathrm{~cm}^{-1}$; HRMS (MultiMode ESI/APCI) 
$m / z:[\mathrm{M}+\mathrm{H}]^{+}$calcd for $\mathrm{C}_{12} \mathrm{H}_{18} \mathrm{ClN}_{2} \mathrm{O}, 241.1108$; found, 241.1092; $[\alpha]^{25} \mathrm{D}+39.4$ (c 0.96, $\mathrm{MeOH},>99 \%$ ee).<smiles>CC(C)(C)[13CH](CCl)NC(=O)c1ccccn1</smiles>

amide chloride $\cdot \mathrm{HCl}$ 11

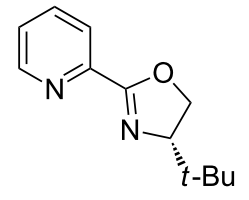

(S)-t-BuPyOx
(S)-4-(tert-butyl)-2-(pyridin-2-yl)-4,5-dihydrooxazole (1): A $500 \mathrm{~mL}$ 3-neck round bottom flask was charged with a stir bar, amide chloride hydrochloric acid salt 11 (10.26 g, $37.0 \mathrm{mmol}$, 1.00 equiv) and $\mathrm{MeOH}(100 \mathrm{~mL})$. To the clear solution was added powdered $\mathrm{NaOMe}(9.99 \mathrm{~g}, 185.0 \mathrm{mmol}, 5.00$ equiv), and the resulting mixture was heated to $55^{\circ} \mathrm{C}$ in an oil bath. The slurry was stirred for $3 \mathrm{~h}$ until the free amide chloride was fully consumed, according to TLC analysis ( $4: 1$ hexanes/acetone). After removing the oil bath, toluene $(100 \mathrm{~mL})$ was added and the mixture was concentrated on a rotary evaporator $\left(40{ }^{\circ} \mathrm{C}, 60 \mathrm{mmHg}\right)$ to remove $\mathrm{MeOH}$. The residual mixture was extracted with $\mathrm{H}_{2} \mathrm{O}(100 \mathrm{~mL})$ and the aqueous phase was back extracted with toluene $(40 \mathrm{~mL} \times 2)$. The combined organic extracts were dried over $\mathrm{Na}_{2} \mathrm{SO}_{4}(10 \mathrm{~g})$, filtered, and concentrated under reduced pressure. The residue was purified by flash column chromatography using American International Chemical ZEOprep ${ }^{\circledR} 60$ ECO 40-63 micron silica gel (4:1 hexanes/acetone) to yield $(S)$ - $t$-BuPyOx (1) as a white solid (5.44 g, 72\% yield). $R_{\mathrm{f}} 0.44$ with $3: 2$ hexanes/acetone; mp 70.2-71.0 ${ }^{\circ} \mathrm{C} ;{ }^{1} \mathrm{H}$ NMR $\left(500 \mathrm{MHz}, \mathrm{CDCl}_{3}\right) \delta 8.71$ (ddd, $J=4.8,1.8,0.9 \mathrm{~Hz}, 1 \mathrm{H}), 8.08(\mathrm{dt}, J=7.9,1.1 \mathrm{~Hz}, 1 \mathrm{H}), 7.77(\mathrm{dt}$, $J=7.7,1.7 \mathrm{~Hz}, 1 \mathrm{H}), 7.37$ (ddd, $J=7.0,4.5,1.0 \mathrm{~Hz}, 1 \mathrm{H}), 4.45$ $(\mathrm{dd}, J=10.2,8.7 \mathrm{~Hz}, 1 \mathrm{H}), 4.31(\mathrm{t}, J=8.5 \mathrm{~Hz}, 1 \mathrm{H}), 4.12(\mathrm{dd}$, $J=10.2,8.5 \mathrm{~Hz}, 1 \mathrm{H}), 0.98(\mathrm{~s}, 9 \mathrm{H}) ;{ }^{13} \mathrm{C} \mathrm{NMR}(125 \mathrm{MHz}$, $\left.\mathrm{CDCl}_{3}\right) \delta 162.4,149.6,147.0,136.5,125.4,124.0,76.5,69.3$, 34.0, 26.0; IR (neat film, NaCl): 2981, 2960, 2863, 1641, 1587, 1466, 1442, 1358, 1273, 1097, 1038, $968 \mathrm{~cm}^{-1}$; HRMS (MultiMode ESI/APCI) $m / z$ : $[\mathrm{M}+\mathrm{H}]^{+}$calcd for $\mathrm{C}_{12} \mathrm{H}_{17} \mathrm{ON}_{2}$, 205.1335; found, 205.1327; $[\alpha]^{25} \mathrm{D}-90.5$ (c 1.15, $\mathrm{CHCl}_{3}$, $>99 \%$ ee).

\section{Supporting Information}

Materials and methods, auxiliary experimental details, and relevant NMR spectra are provided.

\section{Supporting Information File 1}

Experimental details.

[http://www.beilstein-journals.org/bjoc/content/ supplementary/1860-5397-9-187-S1.pdf]

\section{Acknowledgements}

This publication is based on work supported by NIH-NIGMS (R01GM080269-01), and the authors additionally thank Amgen, Abbott, Boehringer Ingelheim, and Caltech for financial support. J.C.H. thanks the American Chemical Society Division of Organic Chemistry for a graduate research fellowship. We also thank Shionogi \& Co., Ltd. for a research grant and fellowship to H.S.

\section{References}

1. Podhajsky, S. M.; Iwai, Y.; Cook-Sneathen, A.; Sigman, M. S. Tetrahedron 2011, 67, 4435-4441. doi:10.1016/j.tet.2011.02.027

2. Aranda, C.; Cornejo, A.; Fraile, J. M.; García-Verdugo, E.; Gil, M. J.; Luis, S. V.; Mayoral, J. A.; Martínez-Merino, V.; Ochoa, Z. Green Chem. 2011, 13, 983-990. doi:10.1039/c0gc00775g

3. Pathak, T. P.; Gligorich, K. M.; Welm, B. E.; Sigman, M. S. J. Am. Chem. Soc. 2010, 132, 7870-7871. doi:10.1021/ja103472a

4. Jiang, F.; Wu, Z.; Zhang, W. Tetrahedron Lett. 2010, 51, 5124-5126. doi:10.1016/j.tetlet.2010.07.084

5. Jensen, K. H.; Pathak, T. P.; Zhang, Y.; Sigman, M. S. J. Am. Chem. Soc. 2009, 131, 17074-17075. doi:10.1021/ja909030c

6. He, W.; Yip, K.-T.; Zhu, N.-Y.; Yang, D. Org. Lett. 2009, 11, 5626-5628. doi:10.1021/ol902348t

7. Dai, H.; Lu, X. Tetrahedron Lett. 2009, 50, 3478-3481. doi:10.1016/j.tetlet.2009.03.005

8. Linder, D.; Buron, F.; Constant, S.; Lacour, J. Eur. J. Org. Chem. 2008, 5778-5785. doi:10.1002/ejoc.200800854

9. Schiffner, J. A.; Machotta, A. B.; Oestreich, M. Synlett 2008, 2271-2274. doi:10.1055/s-2008-1078271

10. Koskinen, A. M. P.; Oila, M. J.; Tois, J. E. Lett. Org. Chem. 2008, 5, 11-16. doi:10.2174/157017808783330216

11. Zhang, Y.; Sigman, M. S. J. Am. Chem. Soc. 2007, 129, 3076-3077. doi:10.1021/ja070263u

12. Yoo, K. S.; Park, C. P.; Yoon, C. H.; Sakaguchi, S.; O’Neill, J.; Jung, K. W. Org. Lett. 2007, 9, 3933-3935. doi:10.1021/ol701584f

13. Dhawan, R.; Dghaym, R. D.; St Cyr, D. J.; Arndtsen, B. A. Org. Lett. 2006, 8, 3927-3930. doi:10.1021/ol061308j

14. Xu, W.; Kong, A.; Lu, X. J. Org. Chem. 2006, 71, 3854-3858. doi:10.1021/jo060288w

15. Malkov, A. V.; Stewart Liddon, A. J. P.; Ramírez-López, P.; Bendová, L.; Haigh, D.; Kočovský, P. Angew. Chem., Int. Ed. 2006, 45, 1432-1435. doi:10.1002/anie.200503941

16. Abrunhosa, I.; Delain-Bioton, L.; Gaumont, A.-C.; Gulea, M.; Masson, S. Tetrahedron 2004, 60, 9263-9272. doi:10.1016/j.tet.2004.07.048

17. Brunner, H.; Kagan, H. B.; Kreutzer, G. Tetrahedron: Asymmetry 2003, 14, 2177-2187. doi:10.1016/S0957-4166(03)00433-6

18. Cornejo, A.; Fraile, J. M.; García, J. I.; Gil, M. J.; Herrerías, C. I.; Legarreta, G.; Martínez-Merino, V.; Mayoral, J. A. J. Mol. Catal. A: Chem. 2003, 196, 101-108. doi:10.1016/S1381-1169(02)00638-6

19. Zhang, Q.; Lu, X.; Han, X. J. Org. Chem. 2001, 66, 7676-7684. doi:10.1021/jo0105181

20. Zhang, Q.; Lu, X. J. Am. Chem. Soc. 2000, 122, 7604-7605. doi:10.1021/ja001379s

21. Perch, N. S.; Pei, T.; Widenhoefer, R. A. J. Org. Chem. 2000, 65, 3836-3845. doi:10.1021/jo0003192 
22. Bremberg, U.; Rahm, F.; Moberg, C. Tetrahedron: Asymmetry 1998, 9 , 3437-3443. doi:10.1016/S0957-4166(98)00346-2

23. Brunner, H.; Obermann, U.; Wimmer, P. Organometallics 1989, 8, 821-826. doi:10.1021/om00105a039

24. Kikushima, K.; Holder, J. C.; Gatti, M.; Stoltz, B. M. J. Am. Chem. Soc. 2011, 133, 6902-6905. doi:10.1021/ja200664x

25. In fact, we have come to accept that the reaction requires a small amount of water to run efficiently. Typically, 5 equiv water are added to each reaction.

26. Brunner, H.; Obermann, U. Chem. Ber. 1989, 122, 499-507. doi:10.1002/cber.19891220318

27. This route, reported by our group in [24], is adapted from the synthesis reported in [26]. See Supporting Information File 1 for experimental details.

28. Jensen, K. H.; Webb, J. D.; Sigman, M. S. J. Am. Chem. Soc. 2010, 132, 17471-17482. doi:10.1021/ja108106h

29. Degradation experiments demonstrate that ligand 1 is susceptible to hydrolysis. Exposure of $t$-BuPyOx to $3 \mathrm{~N} \mathrm{HCl}$ results in complete hydrolysis to amide 4 as observed by ${ }^{1} \mathrm{H}$ NMR.

30. Attempts to isolate $t$-BuPyOx as a salt were successful with $\mathrm{HBF}_{4}$, however the resulting compound was very unstable to atmospheric moisture. See Supporting Information File 1.

\section{License and Terms}

This is an Open Access article under the terms of the Creative Commons Attribution License

(http://creativecommons.org/licenses/by/2.0), which permits unrestricted use, distribution, and reproduction in any medium, provided the original work is properly cited.

The license is subject to the Beilstein Journal of Organic Chemistry terms and conditions:

(http://www.beilstein-journals.org/bjoc)

The definitive version of this article is the electronic one which can be found at: doi:10.3762/bjoc.9.187 